\title{
Factors Related to a Right-Left Difference in Visual Field Defect in the Eyes with Untreated Normal Tension Glaucoma
}

\author{
Haruka Moroi $\mathbb{D}^{1,2}$ Ayako Anraku $\mathbb{D}^{1},{ }^{1}$ Kyoko Ishida $\mathbb{D}^{1},{ }^{1}$ and Goji Tomita $\mathbb{D}^{1}$ \\ ${ }^{1}$ Department of Ophthalmology, Toho University Ohashi Medical Center, 2-17-6 Ohashi, Meguro-ku, Tokyo 153-8515, Japan \\ ${ }^{2}$ Department of Ophthalmology, Kanto Rosai Hospital, 1-1 Kigetsu Sumiyoshi-cho, Nakahara-ku, Kawasaki 211-8510, Japan
}

Correspondence should be addressed to Ayako Anraku; ayakoanraku@gmail.com

Received 28 July 2017; Revised 22 December 2017; Accepted 2 January 2018; Published 15 February 2018

Academic Editor: Paolo Fogagnolo

Copyright ( 2018 Haruka Moroi et al. This is an open access article distributed under the Creative Commons Attribution License, which permits unrestricted use, distribution, and reproduction in any medium, provided the original work is properly cited.

Purpose. To investigate factors related to a right-left difference in visual field defect in untreated normal tension glaucoma (NTG). Methods. The medical records of 92 patients with untreated NTG were reviewed. Ocular blood flow was evaluated with laser speckle flowgraphy, and the mean blur rate (MBR) at the optic nerve head was analyzed. Relationships between right-left differences in mean deviation (MD), intraocular pressure, MBR, spherical equivalent, central corneal thickness, and mean ocular perfusion pressure were evaluated using Spearman's rank correlation coefficient. Multiple regression analysis was used to detect factors contributing to a right-left difference in MD. Results. The right-left difference in MD was correlated with differences in intraocular pressure $(r=-0.263, P=0.011)$, MBR $(r=0.417, P<0.001)$, and spherical equivalent $(r=0.213, P=0.042)$, but not with central corneal thickness or mean ocular perfusion pressure. Multiple regression analysis showed that a difference in MBR was the only significant contributor to a right-left difference in MD (slope $0.047,95 \%$ confidence interval $0.025-0.069$; $P<0.001)$. Conclusion. In untreated NTG, a difference in blood flow at the optic nerve head was a significant contributor to a right-left difference in visual field defect.

\section{Introduction}

Previous studies have reported a correspondence between intraocular pressure (IOP) and greater visual damage when the IOP is not equal between the right and left eyes in patients with normal tension glaucoma (NTG) [1-4]. However, the randomized controlled Low-Pressure Glaucoma Treatment Study of 190 patients with NTG reported that asymmetric IOP was not related to visual field asymmetry [5]. It has also been reported that about $70 \%$ of patients with asymmetric visual field defects do not have unequal mean IOP $[1,2]$. Therefore, factors other than IOP are likely to contribute to development of visual field defects in patients with NTG.

Lee et al. reported that patients with NTG and asymmetric progression showed a decrease in the diameter of the central retinal artery over time in the eye that progressed but not in the eye that remained stable [6]. Further, in a study of retrobulbar hemodynamics in patients with NTG who had asymmetric visual field change and asymmetric ocular perfusion pressure, Kondo et al. found that the flow velocity and resistance index in the ophthalmic artery were significantly higher in subjects with higher ocular perfusion pressure in the eye that had a worse mean deviation (MD) than in subjects with higher ocular perfusion pressure in the eye that had better MD [7]. These results suggest that vascular abnormalities are involved in the development and progression of NTG.

Laser speckle flowgraphy (LSFG) utilizes the laser speckle phenomenon to measure ocular blood flow in a noninvasive manner [8]. LSFG provides the mean blur rate (MBR), a relative measure of blood flow expressed in arbitrary units (AU). Although MBR is not an exact measure, it is proportional to blood velocity and has been used to measure relative differences in blood flow at the optic nerve head $(\mathrm{ONH})$ [9-12]. The MBR of the $\mathrm{ONH}$ has been shown to be strongly correlated with absolute blood flow values 
measured by hydrogen gas clearance or the microsphere method in primates and rabbits [13-15]. Moreover, LSFG was reported to be a reliable method for quantitative assessment of ocular blood flow in Japanese [16] and west European populations [17].

There has been one study that investigated intraindividual (right-left) correlations between the microcirculation at the ONH as measured by scanning laser Doppler flowmetry and visual field parameters in patients with treated primary open-angle glaucoma (POAG) [18]. However, to the best of our knowledge, there have been no reports on the association of an interocular difference in $\mathrm{ONH}$ blood flow with a right-left difference in visual field defect in untreated NTG. Therefore, we investigated the factors associated with a right-left difference in visual field defect in untreated NTG using LSFG.

\section{Materials and Methods}

2.1. Study Subjects. The study protocol was approved by the Toho University Ohashi Medical Center Institutional Review Board (approval number H16061) and adhered to the tenets of the Declaration of Helsinki. We retrospectively reviewed the medical records of patients with glaucoma in whom the ocular circulation was measured by LSFG before initiation of glaucoma treatment at the Toho University Ohashi Medical Center between January 2013 and June 2016. Subjects who met all of the following criteria were included: untreated NTG with normal and open anterior chamber angles on slitlamp biomicroscopy and gonioscopy, an $\mathrm{ONH}$ that appeared to be glaucomatous on stereoscopic evaluation with a corresponding visual field defect, IOP $\leq 21 \mathrm{mmHg}$ throughout 1 day (measured every 3 hours) or on at least three separate days, a spherical refractive error between -8.00 and +4.00 diopters (D), a cylindrical refractive error within $2.5 \mathrm{D}$, and best-corrected visual acuity of at least 20/25. A visual field defect was defined as having three or more significant $(P<0.05)$ nonedge-contiguous points, with at least one point at the $P<0.01$ level in the pattern deviation plot, along with grading outside normal limits on the glaucoma hemifield test. Subjects were excluded from the analysis if they had significant cataract which may influence refractive errors and visual fields, a history of intraocular surgery, intraocular disease (other than NTG), or systemic disease that could affect visual field test results. The IOP was measured with a Goldmann applanation tonometer, and the mean IOP for three separate days was used for the analyses.

Systolic blood pressure (SBP) and diastolic blood pressure (DBP) were measured before performing the LSFG measurements. The mean blood pressure (MBP) and mean ocular perfusion pressure (MOPP) were calculated as follows:

$$
\begin{aligned}
\mathrm{MBP} & =\mathrm{DBP}+\frac{1}{3}(\mathrm{SBP}-\mathrm{DBP}), \\
\mathrm{MOPP} & =\frac{2}{3}(\mathrm{MBP}-\mathrm{IOP}) .
\end{aligned}
$$

2.2. Laser Speckle Flowgraphy. Blood flow at the $\mathrm{ONH}$ was evaluated with LSFG (LSFG-NAVI version 3.1.39.2 software,
Softcare Ltd., Fukuoka, Japan), and the MBR was used as a relative measure of blood flow. The principle and methods of LSFG have been described elsewhere $[8,19]$. Briefly, the instrument is comprised of a fundus camera equipped with a diode laser (wavelength $830 \mathrm{~nm}$ ) and a digital chargecoupled device camera (resolution $750 \times 360$ pixels). The ONH margins were measured with an ellipsoidal band, and the position of the ONH was saved in the system software. The LSFG analysis software automatically calculated the mean MBR in all areas of the optic disc, the mean MBR in the vessel area of the optic disc, and the mean MBR in the tissue area of the optic disc. We used the mean of the MBR values in all areas of the optic disc in this study. The pupils of the eyes enrolled in the study were dilated using $0.4 \%$ tropicamide before LSFG examination, and three consecutive measurements were taken for each subject. The average of the three measurements was used in the analyses.

2.3. Visual Field Analyses. Standard automated perimetry was performed using a Humphrey Field Analyzer (Carl Zeiss Meditec Inc., Dublin, CA, USA) with the 30-2 Swedish Interactive Threshold Algorithm. Visual field tests were considered reliable when fixation losses were $<20 \%$, false positives were $<15 \%$, and false negatives were $<25 \%$. For subset analysis, the study eyes were allocated according to their MD value and mean IOP to an IOP-MD-concordant group (higher mean IOP in the eyes with worse MD) and an IOP-MDdiscordant group (equal or lower mean IOP in the eyes with worse MD). Better MD and worse MD were defined according to the MD values of the visual field test performed within 3 months of the LSFG measurements. Two or more examinations were required to confirm a difference in $\mathrm{MD}$ between the eyes if the interocular difference was within $2 \mathrm{~dB}$. The $\mathrm{dB}$ values were converted to the $1 /$ Lambert linear scale for analysis.

2.4. Statistical Analysis. The Wilcoxon signed-rank test was used to evaluate differences between the eyes in each group of subjects. The Mann-Whitney $U$ test was for the evaluation of interocular differences between the groups. Categorical data were compared using the chi-square test. Spearman's rank correlation coefficients were calculated to evaluate the relationship between right-left differences in ocular parameters. Multiple regression analysis was used to detect factors contributing to a right-left difference in the visual field defect, that is, age, sex, IOP, spherical equivalent (SE), MBR, central corneal thickness (CCT), and MOPP. The data are reported as the mean \pm standard deviation. A $P$ value $<0.05$ was considered to be statistically significant.

\section{Results}

Ninety-two subjects (34 male, 58 female, mean age $54.1 \pm$ $11.9 \mathrm{y}$ ) with untreated NTG were included in the study. The demographic and clinical characteristics of subjects are shown in Table 1. The eye with higher IOP had worse MD in $47(51 \%)$ of the subjects, and the eye with a lower MBR had worse MD in 62 (67\%). A comparison of ocular 
TABLE 1: Demographics and clinical characteristics of subjects.

\begin{tabular}{lc}
\hline Demographic characteristics & \\
Age (years) & $54.1 \pm 11.9$ \\
Sex (male/female) & $34 / 58$ \\
Clinical characteristics & \\
SBP (mmHg) & $122.0 \pm 16.1$ \\
DBP (mmHg) & $71.3 \pm 12.5$ \\
MBP (mmHg) & $88.2 \pm 13.0$ \\
Diabetes mellitus & $1(1 \%)$ \\
Hypertension & $16(17 \%)$ \\
Cardiovascular disease & $6(7 \%)$ \\
\hline
\end{tabular}

The data are given as mean \pm SD; SBP: systolic blood pressure; DBP: diastolic blood pressure; MBP: mean blood pressure.

TABLE 2: Comparison of ocular parameters between the eyes with worse MD and the eyes with better MD $(n=92)$.

\begin{tabular}{lccc}
\hline & $\begin{array}{c}\text { Eyes with worse } \\
\text { MD }\end{array}$ & $\begin{array}{c}\text { Eyes with better } \\
\text { MD }\end{array}$ & $\begin{array}{c}P \\
\text { value }\end{array}$ \\
\hline $\begin{array}{l}\text { Mean IOP } \\
\text { (mmHg) }\end{array}$ & $14.8 \pm 2.3$ & $14.7 \pm 2.3$ & 0.051 \\
SE (diopters) & $-3.77 \pm 3.1$ & $-3.48 \pm 3.0$ & $\mathbf{0 . 0 1 1}$ \\
MD (dB) & $-5.20 \pm 5.2$ & $-2.22 \pm 3.7$ & $<\mathbf{0 . 0 0 1}$ \\
PSD (dB) & $7.52 \pm 4.5$ & $3.85 \pm 3.4$ & $<\mathbf{0 . 0 0 1}$ \\
MBR (AU) & $18.4 \pm 4.1$ & $19.5 \pm 4.8$ & $\mathbf{0 . 0 0 1}$ \\
MOPP (mmHg) & $43.6 \pm 8.6$ & $43.7 \pm 8.6$ & 0.168 \\
CCT $(\mu \mathrm{m})$ & $526.8 \pm 31.3$ & $528.3 \pm 31.5$ & 0.194 \\
\hline
\end{tabular}

The data are given as mean $\pm \mathrm{SD}$; values in bold are statistically significant $(P<0.05)$. AU: arbitrary units; CCT: central corneal thickness; IOP: intraocular pressure; MBR: mean blur rate; MD: mean deviation; MOPP: mean ocular perfusion pressure; PSD: pattern standard deviation; SE: spherical equivalent.

parameters between the eyes with worse MD and those with better MD is provided in Table 2. The eyes with worse MD were more myopic than those with better $\mathrm{MD}$ (mean $\mathrm{SE}$ $-3.77 \pm 3.1 \mathrm{D}$ versus $-3.48 \pm 3.0, P=0.011$ ). Although there was no significant difference in mean IOP between the eyes with worse MD and those with better MD $(14.8 \pm 2.3 \mathrm{mmHg}$ versus $14.7 \pm 2.3 \mathrm{mmHg}, P=0.051$ ), the MBR was significantly lower in the eyes with worse $\mathrm{MD}$ than in those with better MD $(18.4 \pm 4.1 \mathrm{AU}$ versus $19.5 \pm 4.8 \mathrm{AU}, P=0.001)$.

Correlations between the right-left difference in MD and the ocular parameters are shown in Table 3. The right-left difference in MD had significant correlations with the difference in mean IOP $(r=-0.263, P=0.011)$, MBR $(r=0.417$, $P<0.001)$, and SE $(r=0.213, P=0.042)$, but not with the difference in CCT $(r=0.160, P=0.129)$ or MOPP $(r=$ $0.190, P=0.070)$. The scatterplots comparing the right-left difference in MD with the differences in mean IOP, MBR, and SE are shown in Figure 1.

Multiple regression analysis for all 92 subjects, wherein the right-left difference in MD was used as the dependent variable and age, sex, intraocular differences in MBR, and mean IOP, SE, CCT, and MOPP were used as explanatory variables, showed that an intraocular difference in MBR
TABLE 3: Correlation between the right-left difference in mean deviation and ocular parameters $(n=92)$.

\begin{tabular}{lcc}
\hline & $\begin{array}{c}\text { Difference in MD } \\
r\end{array}$ & $P$ value \\
\hline Difference in mean IOP & $\mathbf{- 0 . 2 6 3}$ & $\mathbf{0 . 0 1 1}$ \\
Difference in SE & $\mathbf{0 . 2 1 3}$ & $\mathbf{0 . 0 4 2}$ \\
Difference in MBR & $\mathbf{0 . 4 1 7}$ & $<\mathbf{0 . 0 0 1}$ \\
Difference in CCT & 0.160 & 0.129 \\
Difference in MOPP & 0.190 & 0.070 \\
\hline
\end{tabular}

Values in bold are statistically significant $(P<0.05)$. CCT: central corneal thickness; IOP: intraocular pressure; MBR: mean blur rate; MD: mean deviation; MOPP: mean ocular perfusion pressure; SE: spherical equivalent; $r$ : Spearman's rank correlation coefficient.

was a significant contributor to the right-left difference in MD (slope $0.047, \beta=0.413,95 \%$ confidence interval (CI) $0.025-0.069 ; P<0.001)$.

When the subjects were divided into groups according to $\mathrm{MD}$ and mean IOP for subset analysis, $47(51 \%)$ of the 92 subjects were in the IOP-MD-concordant group (higher mean IOP in the eye with worse MD) and $45(49 \%)$ were in the IOP-MD-discordant group (equal or lower mean IOP in the eye with worse MD). Table 4 shows a comparison of the demographic characteristics and interindividual differences in ocular parameters between the two groups. In the IOP-MD-concordant group, the values for age (56.5 \pm $12.5 \mathrm{y}$ versus $51.3 \pm 11.0 \mathrm{y} ; P=0.027)$ and absolute right-left difference in mean IOP $(0.67 \pm 0.51 \mathrm{mmHg}$ versus $0.42 \pm$ $0.53 \mathrm{mmHg} ; P=0.002)$ were greater than in the IOP-MDdiscordant group.

In the IOP-MD-concordant group $(n=47)$, the rightleft difference in MD was significantly correlated with the differences in mean IOP $(r=-0.763, P<0.001)$ and MBR $(r=0.563, P<0.001)$ but not with the difference in SE $(r=0.225, P=0.129)$. Figure 2 shows the scatterplots comparing the right-left difference in MD with the differences in mean IOP and MBR in the IOP-MD-concordant group. In the IOP-MD-discordant group $(n=45)$, the rightleft difference in MD was significantly correlated with the difference in mean IOP $(r=0.602, P<0.001)$ but not with the difference in MBR $(r=0.187, P=0.219)$ or SE $(r=0.165$, $P=0.279$ ). Figure 3 shows the scatterplots comparing the right-left difference in $\mathrm{MD}$ with the differences in mean IOP and MBR in the IOP-MD-discordant group.

The multiple regression analysis that included subjects in the IOP-MD-concordant group $(n=47)$, wherein the rightleft difference in $\mathrm{MD}$ was used as the dependent variable and age, sex, intraocular differences in MBR, and mean IOP, SE, CCT, and MOPP were used as explanatory variables, showed that the intraocular difference in MBR (slope $0.048, \beta=0.437,95 \%$ CI $0.026-0.071 ; P<0.001)$ and mean IOP (slope $-0.211, \beta=-0.500,95 \%$ CI $-0.298-0.124 ; P<$ $0.001)$ contributed significantly to the right-left difference in $\mathrm{MD}$. In contrast, the multiple regression analysis that included subjects in the IOP-MD-discordant group $(n=45)$ and the same variables found that only the intraocular difference in mean IOP contributed significantly to the right-left 


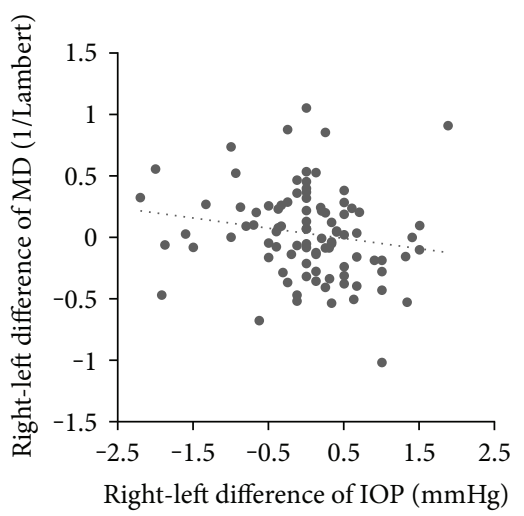

(a)

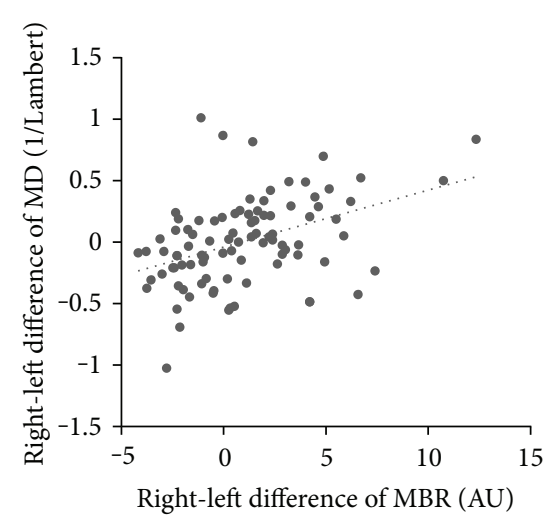

(b)

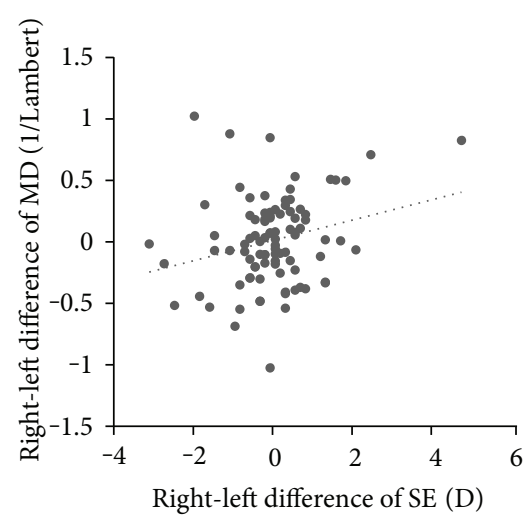

(c)

Figure 1: Scatterplots of right-left difference in MD versus right-left difference in IOP (a), MBR (b), and SE (c) in all 92 subjects. IOP, intraocular pressure; MBR, mean blur rate; $\mathrm{MD}$, mean deviation; and $\mathrm{SE}$, spherical equivalent.

TABLE 4: Comparison of the IOP-MD-concordant group and IOP-MD-discordant group.

\begin{tabular}{lcc}
\hline & $\begin{array}{c}\text { IOP-MD-concordant group } \\
(n=47)\end{array}$ & $\begin{array}{c}\text { IOP-MD-discordant group } \\
(n=45)\end{array}$ \\
\hline Age (years) & $\mathbf{5 6 . 5} \pm \mathbf{1 2 . 5}$ value \\
Sex (male/female) & $15 / 32$ & $\mathbf{5 1 . 3} \pm \mathbf{1 1 . 0}$ \\
MBP (mmHg) & $87.0 \pm 13.4$ & $19 / 26$ \\
Absolute difference in mean IOP (mmHg) & $\mathbf{0 . 6 7} \pm \mathbf{0 . 5 1}$ & $89.3 \pm 12.5$ \\
Absolute difference in SE (diopters) & $0.68 \pm 0.69$ & $\mathbf{0 . 4 2} \pm \mathbf{0 . 5 3}$ \\
Absolute difference in MD (dB) & $3.15 \pm 3.11$ & $0.78 \pm 0.86$ \\
Absolute difference in PSD (dB) & $4.02 \pm 3.45$ & $2.80 \pm 2.61$ \\
Absolute difference in MBR (AU) & $2.45 \pm 2.27$ & $3.54 \pm 3.39$ \\
Absolute difference in CCT $(\mu \mathrm{m})$ & $8.57 \pm 8.96$ & $2.63 \pm 1.99$ \\
\hline
\end{tabular}

Values in bold are statistically significant. AU: arbitrary units; CCT: central corneal thickness; IOP: intraocular pressure; MBP: mean blood pressure; MBR: mean blur rate; MD: mean deviation; PSD: pattern standard deviation; SE: spherical equivalent.

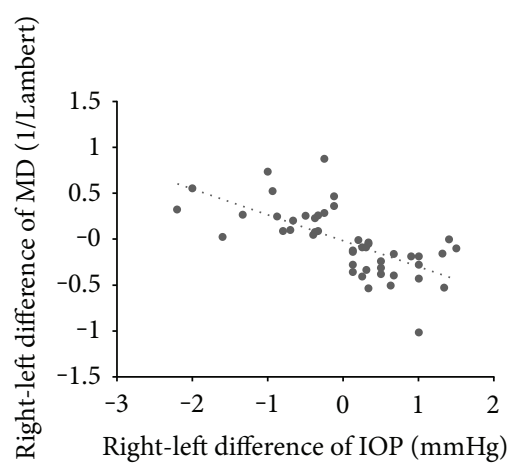

(a)

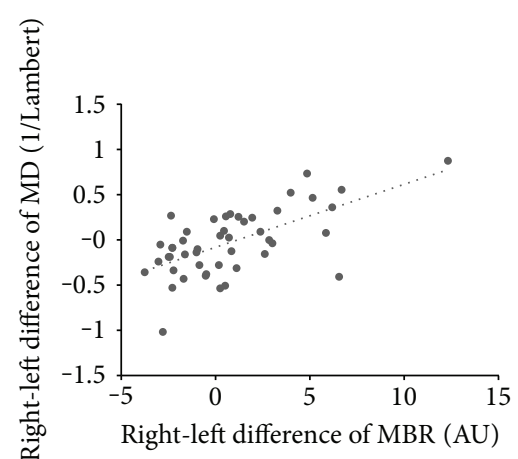

(b)

FIGURE 2: Scatterplots of right-left difference in MD versus right-left difference in IOP (a) and MBR (b) in the IOP-MD-concordant group $(n=47)$. IOP, intraocular pressure; MBR, mean blur rate; and MD, mean deviation.

difference in MD (slope $0.250, \beta=0.476$, 95\% CI 0.108 0.392; $P=0.001)$.

\section{Discussion}

Glaucoma is considered to be a multifactorial disease and is associated with a combination of IOP-dependent and IOP- independent risk factors, including decreased ocular blood flow [10, 20]. Recently, Shiga et al. reported that blood flow at the $\mathrm{ONH}$ was significantly reduced in patients with preperimetric glaucoma (PPG) when compared with that in normal subjects [11]. Their finding is consistent with that in an earlier study using optical coherence tomography (OCT) angiography that reported perfusion of the ONH to 


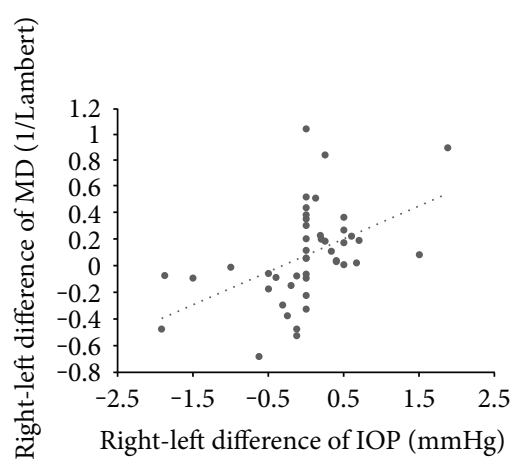

(a)

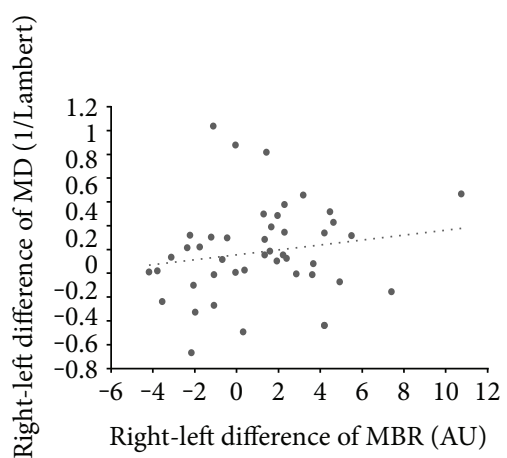

(b)

FIGURE 3: Scatterplots of right-left difference in MD versus right-left difference in IOP (a) and MBR (b) in the IOP-MD-discordant group $(n=45)$. IOP, intraocular pressure; MBR, mean blur rate; and MD, mean deviation.

be significantly lower in patients with PPG than in normal subjects [21]. These findings indicate that blood flow at the $\mathrm{ONH}$ is impaired in the very early stages of glaucoma.

The present study identified a significant correlation between a right-left difference in $\mathrm{ONH}$ blood flow and a difference in the visual field defect in patients with untreated NTG. This result is consistent with that of Ciancaglini et al. [18], who reported that side differences in visual field index were significantly correlated with side differences in the vascular parameters of the lamina cribrosa in treated patients with POAG. Moreover, previous studies have reported that patients with asymmetric glaucomatous visual field loss show asymmetric flow velocities in the central retinal artery and ophthalmic artery [7, 22, 23]. These findings suggest that the pathogenesis of the disease may include vascular abnormalities.

In this study, $51 \%$ of eyes with higher IOP had greater visual field damage, that is, the IOP-MD-concordant group. The subjects in this group were significantly older than those in the IOP-MD-discordant group, and the absolute difference in mean IOP in the IOP-MD-concordant group was greater than that in the IOP-MD-discordant group. These results suggest that advancing age and a greater difference in IOP are associated with asymmetric visual field loss in some patients. Our results are consistent with a previous report showing that $53(60 \%)$ of 88 patients with NTG had more severe visual field defects in the eye with higher IOP than in the fellow eye with lower IOP [4]. However, in the multiple regression analysis that included all subjects, the intraocular difference in IOP was not a significant contributor to the right-left difference in MD. In this study, the mean IOP values recorded on three separate days were used for the analysis and circadian variation in IOP was not evaluated. It is known that variability in nocturnal IOP is an important clinical determinant of the likelihood of progression of visual field defects [24]. Moreover, Kiuchi et al. reported that visual field damage was more severe in eyes with a greater magnitude of IOP elevation in response to postural changes [25]. Besides, some reports showed that IOP elevation asymmetry in lateral decubitus position was associated with asymmetric visual field damage [26-29]. Therefore, it is possible that the mean of IOP values recorded on three separate days may have been insufficient to assess the intraocular difference in IOP.

In the IOP-MD-discordant group, eyes with more visual field damage tended to have lower IOP. We suspected that impaired blood flow at the $\mathrm{ONH}$ might cause this inverse relationship. However, MBR did not contribute to the difference in $\mathrm{MD}$ in this group. Previous studies reported that right-left differences in axial length and disc area were associated with a right-left difference in the visual field defect in patients with NTG [30, 31]. Further, a recent study using swept-source OCT found that the lamina cribrosa was thinner in patients with PPG than in normal subjects [32] and reported that the thickness of the lamina cribrosa was significantly correlated not only with MD but also with the vertical cup-disc ratio. These results suggest that a structural change in the optic nerve contributes to asymmetry of the visual field. In the present study, there was a tendency for the eyes with worse $\mathrm{MD}$ to be more myopic than those with better MD. However, we did not evaluate morphologic changes in the $\mathrm{ONH}$ in all subjects. When we analyzed the data in 60 patients who had OCT data of good quality and matched between structural and functional losses (data are not shown), multiple regression analysis showed that intraocular difference in MBR (slope $0.059, \beta=0.489$, 95\% CI $0.034-$ $0.084 ; P<0.001$ ) and SE (slope $0.111, \beta=0.338,95 \% \mathrm{CI}$ $0.043-0.178 ; \quad P=0.002)$ contributed significantly to the right-left difference in $\mathrm{MD}$.

It has also been reported that asymmetric CCT is associated with visual field asymmetry in patients with POAG [33]. However, in a more recent report by the same group, asymmetric POAG was associated with asymmetric dynamic contour tonometry but not with CCT [34]. Another study reported that visual field asymmetry in POAG was associated with corneal hysteresis but not with CCT [35]. In the present study, an intraocular difference in CCT did not contribute significantly to a right-left difference in MD.

Our study has several limitations. First, its retrospective nature may have introduced a degree of selection bias. Second, as mentioned earlier, we did not evaluate circadian variation in IOP, or effects of postural changes on IOP, so our assessment of the intraocular difference in IOP may have been incomplete. Third, we did not exclude the patients with 
systemic diseases such as diabetes mellitus, hypertension, or cardiovascular disease. Those systemic diseases may cause asymmetric insufficiency in ocular blood flow, which might have influenced on our results. However, when we reanalyzed factors related to a right-left difference in visual field defects in 74 patients without systemic disease, MBR was still detected as the only significant contributor (slope 0.047, $\beta=0.402,95 \%$ CI $0.022-0.072 ; P<0.001)$. Fourth, this study included subjects whose interocular difference in MD was within $2 \mathrm{~dB}$. Although we confirmed the difference in MD between the right eye and left eye on more than two examinations, a small interocular MD difference may be affected by fluctuation of visual fields [36]. Since our subjects were newly diagnosed and untreated NTG patients, subjects with relatively earlier stage of glaucoma were included. It may cause mismatches between structural and functional damage. Assessment of subjects with greater MD asymmetry might have led to different results. However, subjects with an interocular difference in $\mathrm{MD}$ of more than $2 \mathrm{~dB}$ (data not shown) in multiple regression analysis showed that an intraocular difference in MBR contributed significantly to the right-left difference in MD (slope 0.060, $\beta=0.457,95 \%$ CI $0.025-0.095 ; P=0.001$ ). Finally, in this study, we did not evaluate axial lengths, morphologic changes in the $\mathrm{ONH}$, or structural parameters such as retinal nerve fiber layer. In subanalysis for 60 patients who had OCT data and matched between structural and functional losses, multiple regression analysis showed that intraocular difference in MBR (slope $0.059, \beta=0.489,95 \%$ CI 0.034-0.084; $P<$ 0.001 ) and SE (slope $0.111, \beta=0.338,95 \%$ CI $0.043-0.178$; $P=0.002)$ contributed significantly to the right-left difference in MD. Recruitments of more subjects with greater $\mathrm{MD}$ asymmetry and assessment of structural damage are needed as a further research.

\section{Conclusion}

In this study, we demonstrated that a difference in blood flow at the $\mathrm{ONH}$ contributed significantly to the right-left difference in visual field defect in patients with untreated NTG. This suggests that eyes with more visual field damage have a greater reduction in $\mathrm{ONH}$ blood flow. It is uncertain whether the decrease in ONH blood flow is a primary or secondary event caused by glaucomatous optic neuropathy; however, impaired $\mathrm{ONH}$ blood flow has a possible role in the pathogenesis of NTG.

\section{Disclosure}

This study was partially presented at the 7 th World Glaucoma Congress in Helsinki on June 30, 2017.

\section{Conflicts of Interest}

The authors declare that there is no conflict of interest regarding publication of this paper.

\section{Acknowledgments}

The authors are very grateful to Mr. Takashi Sato for the acquisition of the LSFG data.

\section{References}

[1] I. O. Haefliger and R. A. Hitchings, "Relationship between asymmetry of visual field defects and intraocular pressure difference in an untreated normal (low) tension glaucoma population," Acta Ophthalmologica, vol. 68, no. 5, pp. 564-567, 1990.

[2] A. Crichton, S. M. Drance, G. R. Douglas, and M. Schulzer, "Unequal intraocular pressure and Its relation to asymmetric visual field defects in low-tension glaucoma," Ophthalmology, vol. 96, no. 9, pp. 1312-1314, 1989.

[3] M. J. Cartwright and D. R. Anderson, "Correlation of asymmetric damage with asymmetric intraocular pressure in normal-tension glaucoma (low-tension glaucoma)," Archives of Ophthalmology, vol. 106, no. 7, pp. 898-900, 1988.

[4] K. Nakamoto, N. Yasuda, and T. Fukuda, "Correlation of age and intraocular pressure with visual field damage in patients with normal-tension glaucoma," Nippon Ganka Gakkai Zasshi, vol. 112, no. 4, pp. 371-375, 2008.

[5] D. S. Greenfield, J. M. Liebmann, R. Ritch, T. Krupin, and Low-Pressure Glaucoma Study Group, "Visual field and intraocular pressure asymmetry in the low-pressure glaucoma treatment study," Ophthalmology, vol. 114, no. 3, pp. 460465, 2007.

[6] T. E. Lee, Y. Y. Kim, and C. Yoo, "Retinal vessel diameter in normal-tension glaucoma patients with asymmetric progression," Graefe's Archive for Clinical and Experimental Ophthalmology, vol. 252, no. 11, pp. 1795-1801, 2014.

[7] Y. Kondo, Y. Niwa, T. Yamamoto, A. Sawada, A. Harris, and Y. Kitazawa, "Retrobulbar hemodynamics in normal-tension glaucoma with asymmetric visual field change and asymmetric ocular perfusion pressure," American Journal of Ophthalmology, vol. 130, no. 4, pp. 454-460, 2000.

[8] T. Sugiyama, M. Araie, C. E. Riva, L. Schmetterer, and S. Orgul, "Use of laser speckle flowgraphy in ocular blood flow research," Acta Ophthalmologica, vol. 88, no. 7, pp. 723-729, 2010.

[9] N. Aizawa, H. Kunikata, Y. Shiga, Y. Yokoyama, K. Omodaka, and T. Nakazawa, "Correlation between structure/function and optic disc microcirculation in myopic glaucoma, measured with laser speckle flowgraphy," BMC Ophthalmology, vol. 14, p. 113, 2014.

[10] T. Nakazawa, "Ocular blood flow and influencing factors for glaucoma," Asia-Pacific Journal of Ophthalmology, vol. 5, no. 1, pp. 38-44, 2016.

[11] Y. Shiga, H. Kunikata, N. Aizawa et al., "Optic nerve head blood flow, as measured by laser speckle flowgraphy, is significantly reduced in preperimetric glaucoma," Current Eye Research, vol. 41, pp. 1447-1453, 2016.

[12] A. Anraku, K. Ishida, N. Enomoto et al., "Association between optic nerve head microcirculation and macular ganglion cell complex thickness in eyes with untreated normal tension glaucoma and a hemifield defect," Journal of Ophthalmology, vol. 2017, Article ID 3608396, 9 pages, 2017.

[13] L. Wang, G. A. Cull, C. Piper, C. F. Burgoyne, and B. Fortune, "Anterior and posterior optic nerve head blood flow in nonhuman primate experimental glaucoma model measured by laser 
speckle imaging technique and microsphere method," Investigative Ophthalmology \& Visual Science, vol. 53, no. 13, pp. 303-309, 2012.

[14] N. Aizawa, F. Nitta, H. Kunikata et al., "Laser speckle and hydrogen gas clearance measurements of optic nerve circulation in albino and pigmented rabbits with or without optic disc atrophy," Investigative Ophthalmology \& Visual Science, vol. 55, no. 12, pp. 7991-7996, 2014.

[15] H. Takahashi, T. Sugiyama, H. Tokushige et al., "Comparison of CCD-equipped laser speckle flowgraphy with hydrogen gas clearance method in the measurement of optic nerve head microcirculation in rabbits," Experimental Eye Research, vol. 108, pp. 10-15, 2013.

[16] N. Aizawa, Y. Yokoyama, N. Chiba et al., "Reproducibility of retinal circulation measurements obtained using laser speckle flowgraphy-NAVI in patients with glaucoma," Clinical Ophthalmology, vol. 5, pp. 1171-1176, 2011.

[17] N. Luft, P. A. Wozniak, G. C. Aschinger et al., "Ocular blood flow measurements in healthy white subjects using laser speckle flowgraphy," PLoS One, vol. 11, no. 12, article e0168190, 2016.

[18] M. Ciancaglini, P. Carpineto, C. Costagliola, and L. Matropasqua, "Perfusion of the optic nerve head and visual field damage in glaucomatous patients," Graefe's Archive for Clinical and Experimental Ophthalmology, vol. 239, no. 8, pp. 549-555, 2001.

[19] Y. Tamaki, M. Araie, E. Kawamoto, S. Eguchi, and H. Fujii, "Non-contact, two-dimensional measurement of tissue circulation in choroid and optic nerve head using laser speckle phenomenon," Experimental Eye Research, vol. 60, no. 4, pp. 373-383, 1995.

[20] A. P. Cherecheanu, G. Garhofer, D. Schmidl, R. Werkmeister, and L. Schmetterer, "Ocular perfusion pressure and ocular blood flow in glaucoma," Current Opinion in Pharmacology, vol. 13, no. 1, pp. 36-42, 2013.

[21] Y. Jia, J. C. Morrison, J. Tokayer et al., "Quantitative OCT angiography of optic nerve head blood flow," Biomedical Optics Express, vol. 3, no. 1, pp. 3127-3137, 2012.

[22] M. T. Nicolela, S. M. Drance, S. J. Rankin, A. R. Buckley, and B. E. Walman, "Color Doppler imaging in patients with asymmetric glaucoma and unilateral visual field loss," American Journal of Ophthalmology, vol. 121, no. 5, pp. 502-510, 1996.

[23] N. Plange, M. Kaup, O. Arend, and A. Remky, "Asymmetric visual field loss and retrobulbar haemodynamics in primary open-angle glaucoma," Graefe's Archive for Clinical and Experimental Ophthalmology, vol. 244, no. 8, pp. 978-983, 2006.

[24] J. H. Liu, X. Zhang, D. F. Kripke, and R. N. Weinreb, "Twentyfour-hour intraocular pressure pattern associated with early glaucomatous changes," Investigative Ophthalmology \& Visual Science, vol. 44, no. 4, pp. 1586-1590, 2003.

[25] T. Kiuchi, Y. Motoyama, and T. Oshika, "Postural response of intraocular pressure and visual field damage in patients with untreated normal-tension glaucoma," Journal of Glaucoma, vol. 19, no. 3, pp. 191-193, 2010.

[26] K. N. Kim, J. W. Jeoung, K. H. Park, D. S. Lee, and D. M. Kim, "Effect of lateral decubitus position on intraocular pressure in glaucoma patients with asymmetric visual field loss," Ophthalmology, vol. 120, no. 4, pp. 731-735, 2013.

[27] K. N. Kim, J. W. Jeoung, K. H. Park, D. M. Kim, and R. Ritch, "Relationship between preferred sleeping position and asymmetric visual field loss in open-angle glaucoma patients,"
American Journal of Ophthalmology, vol. 157, no. 3, pp. 739$745,2014$.

[28] J. Y. Lee, C. Yoo, and Y. Y. Kim, “The effect of lateral decubitus position on intraocular pressure in patients with untreated open-angle glaucoma," American Journal of Ophthalmology, vol. 155, no. 2, pp. 329-335, 2013.

[29] C. Yoo, S. Lin, K. Na, E. J. Yoo, Y. M. Choi, and Y. Y. Kim, "Habitual sleeping position and asymmetric structural and functional loss in patients with open angle glaucoma," Acta Ophthalmologica, vol. 93, no. 7, pp. e593-e595, 2015.

[30] F. Hayamizu, Y. Yamazaki, and T. Nakagami, "Investigation of the correlation between the right-left differences of visual field defects and the right-left differences of ocular anatomical factors in patients with normal-tension glaucoma," Nippon Ganka Gakkai Zasshi, vol. 115, no. 4, pp. 362-367, 2011.

[31] G. Tomita, K. Nyman, C. Raitta, and M. Kawamura, "Interocular asymmetry of optic disc size and its relevance to visual field loss in normal-tension glaucoma," Graefe's Archive for Clinical and Experimental Ophthalmology, vol. 232, no. 5, pp. 290-296, 1994.

[32] K. Omodaka, S. Takahashi, A. Matsumoto et al., "Clinical factors associated with lamina cribrosa thickness in patients with glaucoma, as measured with swept source optical coherence tomography," PLoS One, vol. 11, no. 4, article e0153707, 2016.

[33] M. Sullivan-Mee, J. M. Gentry, and C. Qualls, "Relationship between asymmetric central corneal thickness and glaucomatous visual field loss within the same patient," Optometry and Vision Science, vol. 83, no. 7, pp. 516-519, 2006.

[34] M. Sullivan-Mee, K. D. Halverson, and C. Qualls, "Clinical comparison of Pascal dynamic contour tonometry and goldmann applanation tonometry in asymmetric open-angle glaucoma," Journal of Glaucoma, vol. 16, no. 8, pp. 694699, 2007.

[35] A. Anand, C. G. De Moraes, C. C. Teng, C. Tello, J. M. Liebmann, and R. Ritch, "Corneal hysteresis and visual field asymmetry in open angle glaucoma," Investigative Ophthalmology \& Visual Science, vol. 51, no. 12, article 6514, 2010.

[36] P. Fogagnolo, C. Sangermani, F. Oddone et al., "Long-term perimetric fluctuation in patients with different stages of glaucoma," British Journal of Ophthalmology, vol. 95, no. 2, pp. 189-193, 2011. 


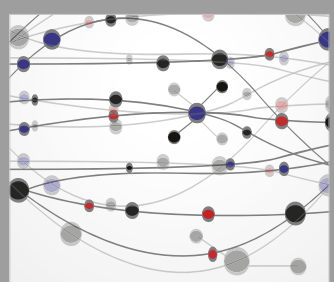

The Scientific World Journal
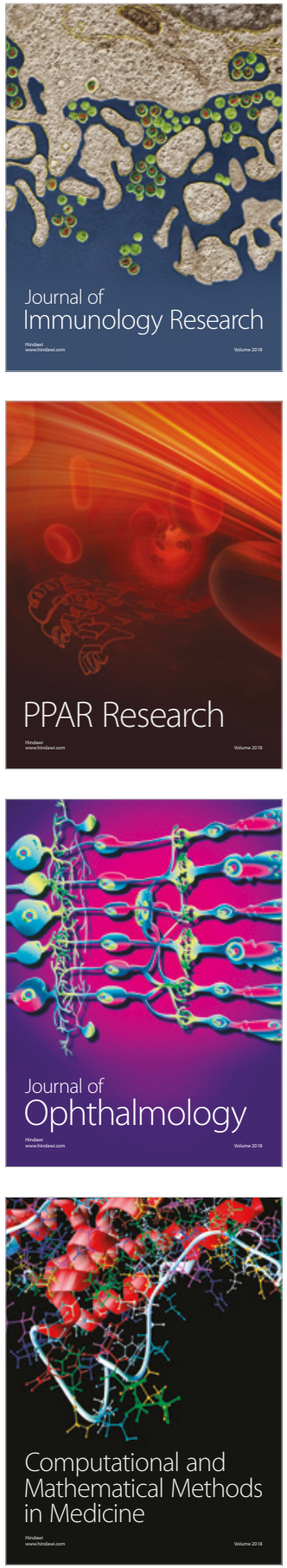

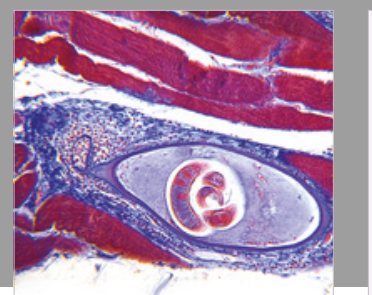

Gastroenterology Research and Practice

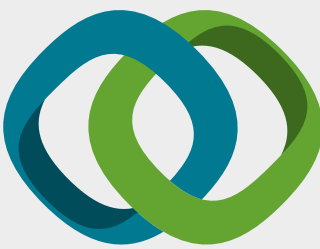

\section{Hindawi}

Submit your manuscripts at

www.hindawi.com
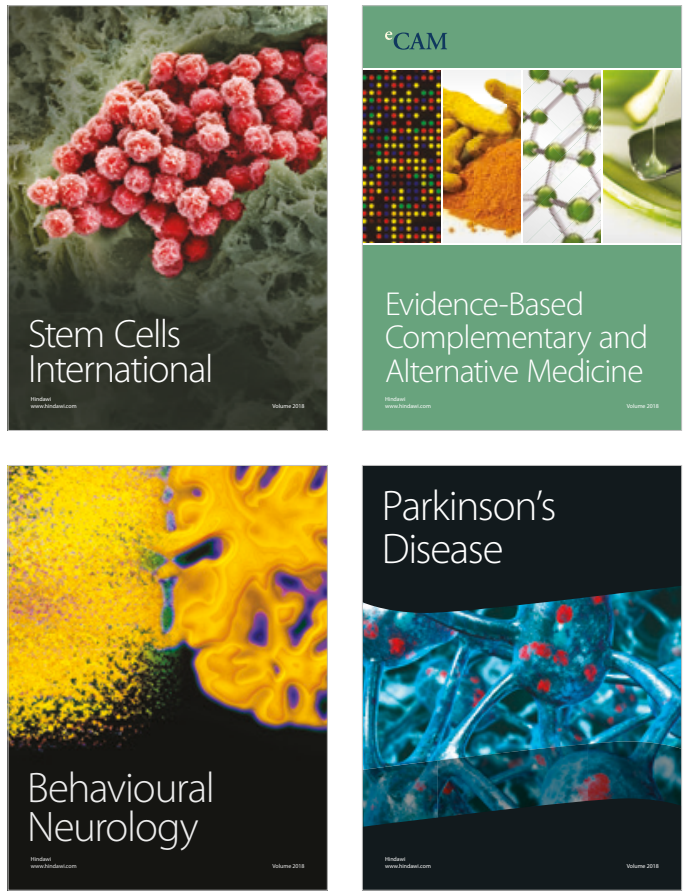

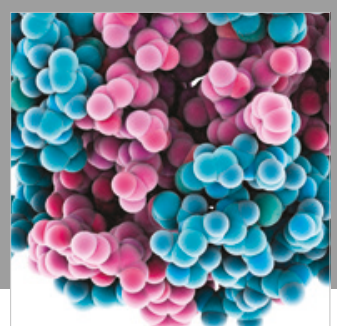

ournal of

Diabetes Research

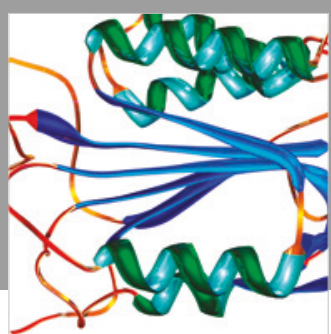

Disease Markers
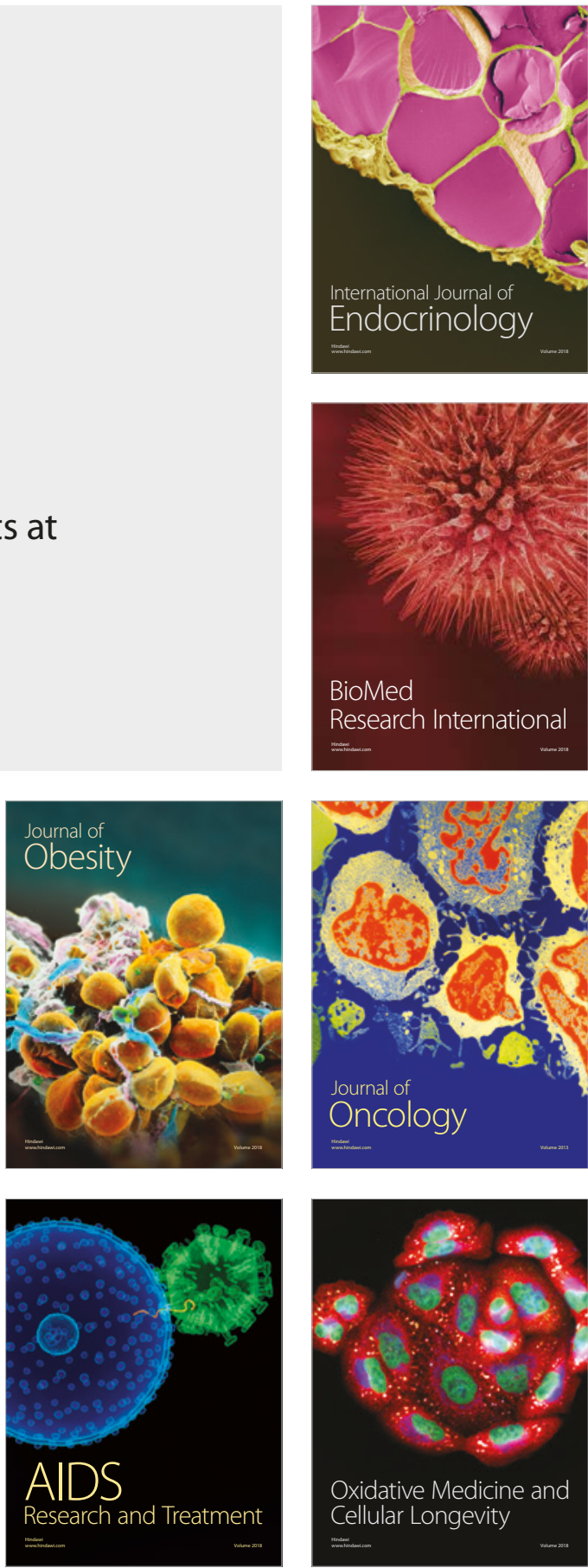